\title{
Effects of Leading Edge Sweep on the Cavitating Characteristics of Inducer Pumps
}

\author{
Allan J. Acosta, ${ }^{1}$ Yoshinobu Tsujimoto, ${ }^{2}$ Yoshiki Yoshida, ${ }^{2}$ Seiji Azuma, ${ }^{2}$ \\ and Paul Cooper ${ }^{3}$ \\ ${ }^{1}$ California Institute of Technology, Mechanical Engineering, Pasadena, California, USA; \\ ${ }^{2}$ Osaka University, Engineering Science, Osaka, Japan; ${ }^{3}$ Fluid Machinery Research, Inc., \\ Titusville, New Jersey, USA
}

It is well known that leading edge sweep has a favorable effect on the cavitation of turbomachines. However, the mechanisms of the improvement have not been made clear. It has been shown that the lift and the drag on a cavitating swept single hydrofoil can be correlated fairly well based on the velocity component normal to the leading edge. In the present paper, such correlations for swept cascades are derived and the results are examined, neglecting the full geometrical effects of the inducer rotor. It is shown that the correlations can simulate the developments of various types of cavitation, including alternate blade cavitation, rotating cavitation, and cavitation surge. This result is based on the observation that the steady cavity length, as well as the developments of various types of cavitation, is fairly well predicted by the correlation.

Keywords Inducer, Cavitation, Leading edge sweep, Cross flow plane

\section{INTRODUCTION}

The effect of inclining the leading edge of a wing to the oncoming flow, called sweep, is familiar to us all from seeing modern high-speed aircraft. Sweep is usually backwards (the angle between the leading edge and the flow is less than $90^{\circ}$ ), but it can be forward as well. The primary effect of sweep in aerodynamics

Received in final form on 10 July 2001.

This study was triggered by a discussion at US-Japan Seminar sponsored by NSF and JSPS. Experimental results were obtained under the supports of SNECMA, division SEP and the Ministry of Education, Japan. These supports are gratefully acknowledged.

Address correspondence to Allan J. Acosta, California Institute of Technology, Mechanical Engineering, Pasadena, CA 91125. E-mail: allan@busumann.thomas.caltech.edu is to forestall the influence of compressibility. Similar problems occur in gas turbine blading described in detail by Wadia et al. (1998). But in normal industrial practice, the leading edges of conventional pumps often tend to be normal to the oncoming streamlines as seen in a meridional section, i.e., the view of the impeller blades projected onto a radial plane. In that case, we would say there is no sweep; this is a common feature of many pumps. The occurrence of cavitation in liquids causes special problems for pumps and, in many cases, the inlet of a pump is fitted with a special rotor to operate satisfactorily with extensive cavitation and it is usually called an inducer pump ${ }^{1}$. This rotor may be an integral part of the main pump to follow or a separate device. Typically, this is an unshrouded rotor with small tip clearance with, as a result, cantilever-mounted blades. Cavitation is a notoriously unsteady phenomenon resulting in highlyfluctuating blade loadings and, as a consequence, most designers have intuitively inclined the blade leading edge backwards (in the meridional plane) to minimize structural and vibration loads on the unsupported leading edge. But there also can be consequences for cavitation performance, as many designers have noticed that such swept-back leading edges are able to operate an lower inlet pressures than radial ones, thereby achieving a lower cavitation number or a higher suction specific speed. In fact, one of us (Cooper, 1973) has patented an impeller design in which the leading edge in the meridional view (see Fig. 1b) is highly inclined backwards - or forwards with the blade elements similarly inclined. Most inducer pumps, however, traditionally have blade elements (or blade generators) that are radial or nearly so. Thus, these blades are somewhat like that of a conventional wing. Interestingly enough, experimental measurements on a single foil in cavitating flow have been made (Ihara et al., 1989) in which a simple scaling rule, the same as for compressible flow, collapses the cavitating performance data as a function of

\footnotetext{
${ }^{1}$ See, e.g., the article "Inducer Pumps-An Apercu," ISROMAC IV, section $B$, page 1 for a recent review of these pumps.
} 
(a) The end view

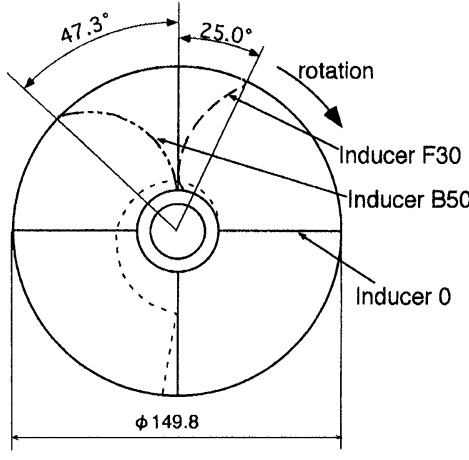

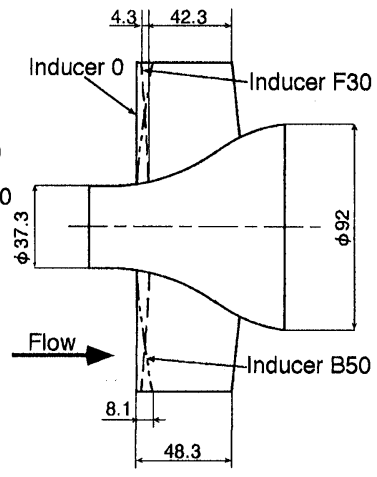

(b) The meridional view
FIGURE 1

Geometry of leading edges of test inducers. (The leading edge profiles of F30 and B50 are shown in (a) only for one blade.)

sweep angle surprisingly well. They find that (from their conclusions) the lift drag ratio for highly swept foils is superior to the unswept case at the same and even lower cavitation number (all at constant angle of attack) and that the flow velocity normal to the foil should be selected to calculate a cavitation number.

With this background, we want to propose similar rules for inducer pumps based on the idea of a cascade of straight blades simulating the flow in the tip region of an inducer of radial blade elements with an axial inflow. The leading edges of the blades in the meridional view can be inclined backwards or forwards as shown in Fig. 1(b). The end view of the inducer shown in Fig. 1(a) shows the leading edge swept backwards or forwards but subtending a relatively large angle.

\section{GEOMETRY OF THE SWEPT CASCADE}

The cascade is shown in Fig. 2; several views are shown which are needed for clarity. The upper or plan view of the cascade shows straight uncambered blades spaced $s$ apart along the cascade axis (the plane normal to the inducer axis in Fig. 1(b)). The blades are inclined at blade angle $\beta$ with respect to this axis. The leading edges of these blades are shown in the meridional view inclined at angle $\delta$ from what would be a radial line in Fig. 1(b). Let us select two points on one blade, $O$ and $A$. Corresponding points in the meridional plane lie along $l-l$ spanning a vertical height $\eta_{s}$ (in the radial direction). These points and at the next blade $O^{\prime}, A^{\prime}$ are observed in the true view of the blade leading edge defined by cut $B-B$. The bold line $O A$ is the true view of the leading edge. This line makes an angle $\lambda$ to the plane of the inducer axis, namely, the projection of $O-O^{\prime}$. The adjacent blade in this true view is $O^{\prime}-A^{\prime}$. The plane normal to $O A, O^{\prime} A^{\prime}$ and to the plane of the paper is the cross flow plane. Note that the line $O^{\prime} A^{\prime}$ is hidden from view by the first blades. Now in the true view plane, $B B$, project a normal to $O A$ from $O^{\prime}$ ending at $P$, a point on the leading edge of blade $B_{1}$. Point $P$ also appears in the plan view on the leading edge of blade $B_{1}$. Imagine now we progress from $P$ normal to the leading edge along blade $B_{1}$

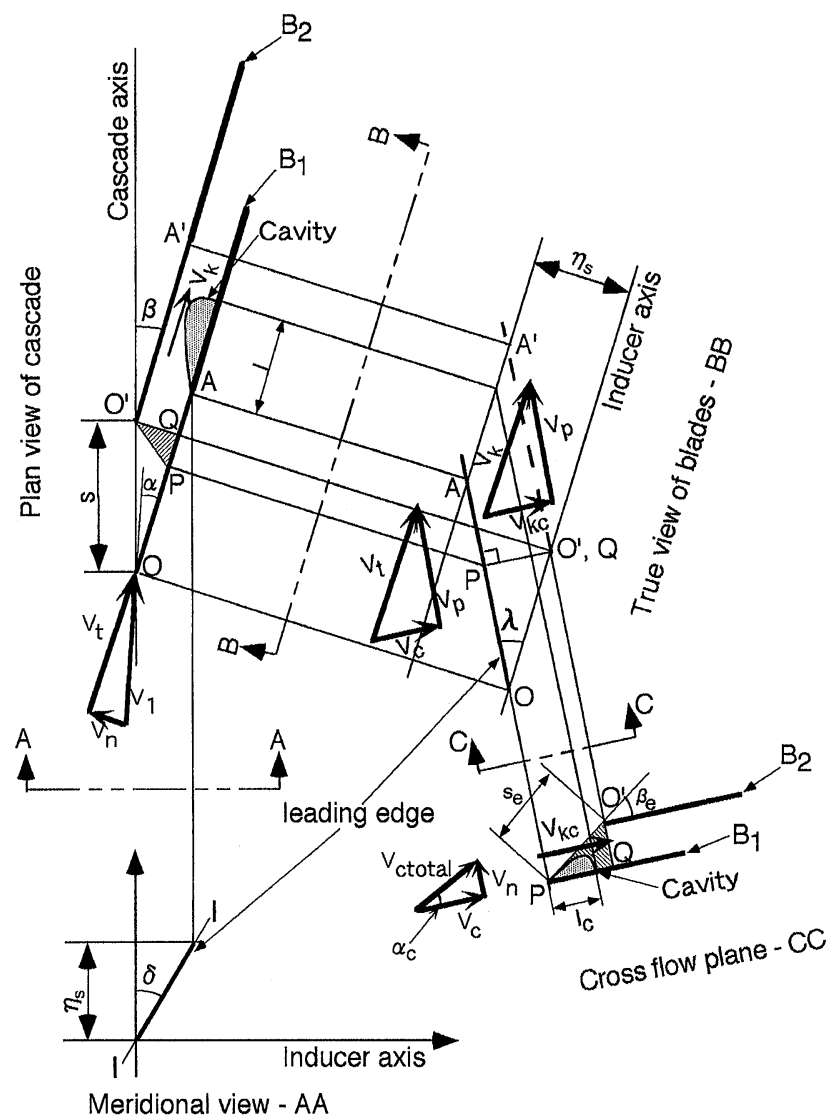

FIGURE 2

Geometry of swept cascade.

in the cross flow plane, $C C$, until we are underneath the normal to the next blade, $B_{2}$, at point $Q$. From $Q$ we move to point $O^{\prime}$ on blade $B_{2}$ seen in the plan view. The cross flow plane in the plan view may be identified by the points $P, Q, O^{\prime}$ (a portion of it is shown, cross-hatched for clarity).

We will be concerned with the flow velocities and the effective cascade geometry in this cross-flow plane. Before completing the definitions of the cross-flow cascade geometry, let us describe the velocity components. The velocity approaching the inducer seen in Fig. 1(b) is presumed to be purely axial; relative to the rotating inducer the flow speed is $V_{1}$ and is inclined to the blades with incidence angle $\alpha$ shown in the plan view of Fig. 2. This velocity vector lies in the plane of the inducer in Fig. 2. There is a component of $V_{1}$ that is normal to the cascade blade, $V_{n}$, and a component tangential to the blade surface $V_{t}$ also shown in Fig. 2. The tangential component is resolved into component $V_{c}$ normal to the true view of the leading edge and $V_{p}$ parallel to it. We see in the cross-flow plane (Fig. 2, section $C C$ ) component $V_{c}$ approaching a cascade but one characterized by a new spacing, $s_{e}$, and a new blade angle, $\beta_{e}$. The normal distance between the blades $B_{1}, B_{2}$ shown in the cross-flow and plan view is of course the same, i.e.,

$$
s \sin \beta=s_{e} \sin \beta_{e}, \text { or } \beta_{c}=\sin ^{-1}\left(\left(s / s_{e}\right) \sin \beta\right) .
$$


The sweep angle $\lambda$ is constructed in the true view plane from

$$
\tan \lambda=\frac{\eta_{s}}{\left(\eta_{s} \tan \delta / \sin \beta\right)}, \text { or } \lambda=\tan ^{-1}\left(\frac{\sin \beta}{\tan \delta}\right) .
$$

The effective spacing is determined from its projection $P-O^{\prime}$ in the true view plane and the normal distance above to get

$$
s_{e}=\sqrt{(s \cos \beta \sin \lambda)^{2}+(s \sin \beta)^{2}} .
$$

Note that $s_{e} \leq s$. The true thickness of the blades is $t$; the ratio $t / s$ is an important geometric parameter governing cavitation. Clearly then the effective thickness-spacing ratio $(t / s)_{\mathrm{eff}}$ is

$$
\left(\frac{t}{s}\right)_{\mathrm{eff}}=\left(\frac{t}{s}\right) \bullet\left(\frac{s}{s_{e}}\right) \geq\left(\frac{t}{s}\right) .
$$

Thus the cross-flow geometry is blunter than the normal flow. For a leading edge in the shape of a wedge of included angle $\theta$, it follows that

$$
\theta_{e}=\tan ^{-1}\left(\frac{\tan \theta}{\sin \lambda}\right)
$$

If, as is the case with many small commercial inducers with leading edges machined on a lathe, $\theta=\beta$. Finally, there is the flow incidence angle in the cross flow plane for which

$$
\alpha_{c}=\tan ^{-1}\left(V_{n} / V_{c}\right), \text { or } \alpha_{c}=\tan ^{-1}(\tan \alpha / \sin \lambda),
$$

so that $\alpha_{c} \geq \alpha$. This completes all the geometrical features in the cross-flow plane.

\section{CAVITATION SCALING}

Let the pressure in the cavity be $p_{v}$ and the velocity there be $V_{k}$. Then in the usual way from the Bernoulli Equation, i.e.,

$$
\frac{p_{t 1}}{\rho}=\frac{p_{1}}{\rho}+\frac{1}{2} V_{1}^{2}=\frac{p_{v}}{\rho}+\frac{1}{2} V_{k}^{2},
$$

we define the cavitation number

$$
k=\frac{p_{1}-p_{v}}{\rho V_{1}^{2} / 2}
$$

and so we can say

$$
V_{k}=V_{1} \sqrt{1+k}
$$

in the physical plane. The total velocity approaching the cascade in the cross-flow plane is

$$
V_{c_{\text {total }}}=\sqrt{V_{c}^{2}+V_{n}^{2}}
$$

and, based on this velocity, we can define a cross-flow cavitation number as

$$
k_{c}=\left(p_{1}-p_{v}\right) /\left(\rho V_{c_{\text {total }}}^{2} / 2\right) .
$$

And on the cavity we will have

$$
V_{k c}=V_{c_{\text {total }}} \sqrt{1+k_{c}}
$$

These definitions must make the true velocity on the cavity precisely the same requiring that

$V_{k}^{2}=V_{k c}^{2}+V_{p}^{2}$ or $V_{1}^{2}(1+k)=\left(V_{c}^{2}+V_{n}^{2}\right)\left(1+k_{c}\right)+V_{p}^{2}$.

After substituting these definitions we have the simple result

$$
k_{c}=k /\left(\cos ^{2} \alpha \sin ^{2} \lambda+\sin ^{2} \alpha\right) .
$$

Note that if $\lambda \rightarrow \pi / 2$ (no sweep), $k \rightarrow k_{c}$. For typically small incidence angles $\alpha<<1$,

$$
k_{c}=\left(k / \sin ^{2} \lambda\right)\left(1-0\left(\alpha^{2}\right)\right) \approx k / \sin ^{2} \lambda,
$$

which is the relation we will use. The equivalent formula for a swept-isolated wing quoted by Ihara et al. is (in our notation)

$$
k_{c}=k / \sin ^{2} \lambda
$$

the slight difference arises because our cross-flow velocity is parallel to the chord.

The effect of sweep appears through two mechanisms. One is through the change of cascade geometry in cross flow as shown by Eqs. (3)-(6) obtained in the preceding section. The other is through the cavitation scaling as shown by Eq. (16). It has been shown by two-dimensional linear analysis such as Acosta (1955) that the cavity length, and hence the cavity development, is a function of $k / 2 \alpha$. If we combine Eq. (6) and Eq. (16), we obtain

$$
k_{c} / 2 \alpha_{c}=\frac{k / 2 \alpha}{\sin \lambda} .
$$

If we increase the leading edge sweep, $\lambda$ is decreased. Then $k_{c} / 2 \alpha_{c}$ is increased and hence the cavity length $1 / \mathrm{s}=\left(1_{\mathrm{c}} / \mathrm{s}_{\mathrm{e}}\right)$ $\left(\cos \beta / \cos \beta_{e}\right)$ is decreased. This can be the major reason why the cavitation performance is increased by simply sweeping the leading edge.

\section{COMPARISONS WITH EXPERIMENTS AND THEORETICAL CALCULATIONS}

In order to study the effect of leading edge sweep, systematic experiments were carried out at Osaka University under the support of SNECMA, division SEP. Three inducers tested here have helical blades with the same camber line with straight part near the leading edge. Forward and backward swept inducers 
TABLE I

Geometry of test inducers

\begin{tabular}{lccc}
\hline \multicolumn{1}{c}{ Inducer } & 0 & B50 & F30 \\
\hline \multicolumn{1}{c}{ Sweep } & Without & $\begin{array}{c}\text { Backward } \\
\text { sweep }\end{array}$ & $\begin{array}{c}\text { forward } \\
\text { sweep }\end{array}$ \\
& sweep & $47.3 \mathrm{deg}$. & $25.0 \mathrm{deg}$. \\
\hline Sweep angle $\lambda$ at tip & $90 \mathrm{deg}$. & $35.4 \mathrm{deg}$. & $49.2 \mathrm{deg}$. \\
Number of blades & 4 & 4 & 4 \\
Tip diameter, D1 & $149.8 \mathrm{~mm}$ & $149.8 \mathrm{~mm}$ & $149.8 \mathrm{~mm}$ \\
Inlet tip blade angle, $\beta_{t 1}$ & $7.5 \mathrm{deg}$. & $7.5 \mathrm{deg}$. & $7.5 \mathrm{deg}$. \\
Outlet tip blade angle, $\beta_{t 2}$ & $9.0 \mathrm{deg}$. & $9.0 \mathrm{deg}$. & $9.0 \mathrm{deg}$. \\
Hub/tip ratio at inlet & 0.25 & 0.25 & 0.25 \\
Hub/tip ratio at outlet & 0.51 & 0.51 & 0.51 \\
Solidity at tip & 2.97 & 2.44 & 2.86 \\
Tip clearance & $0.5 \mathrm{~mm}$ & $0.5 \mathrm{~mm}$ & $0.5 \mathrm{~mm}$ \\
Design flow coefficient, $\phi_{d}$ & 0.078 & 0.078 & 0.078 \\
\hline
\end{tabular}

were produced by cutting back the straight part of an unswept inducer so that the inlet blade angle is not changed. Thus, all of the inducers have the same inlet and outlet blade angle. Dimensions of the inducers are shown in Table I. The basic design is the same as for the LE-7 LOX turbopump inducer except that three blades are employed for LE-7. Inducer 0 is without sweep and has a straight radial leading edge. Inducer B50 is produced by cutting back the leading edge by about $47.3^{\circ}$ as shown in Fig. 1 . Inducer F30 is produced by offsetting the leading edge by $35^{\circ}$ at

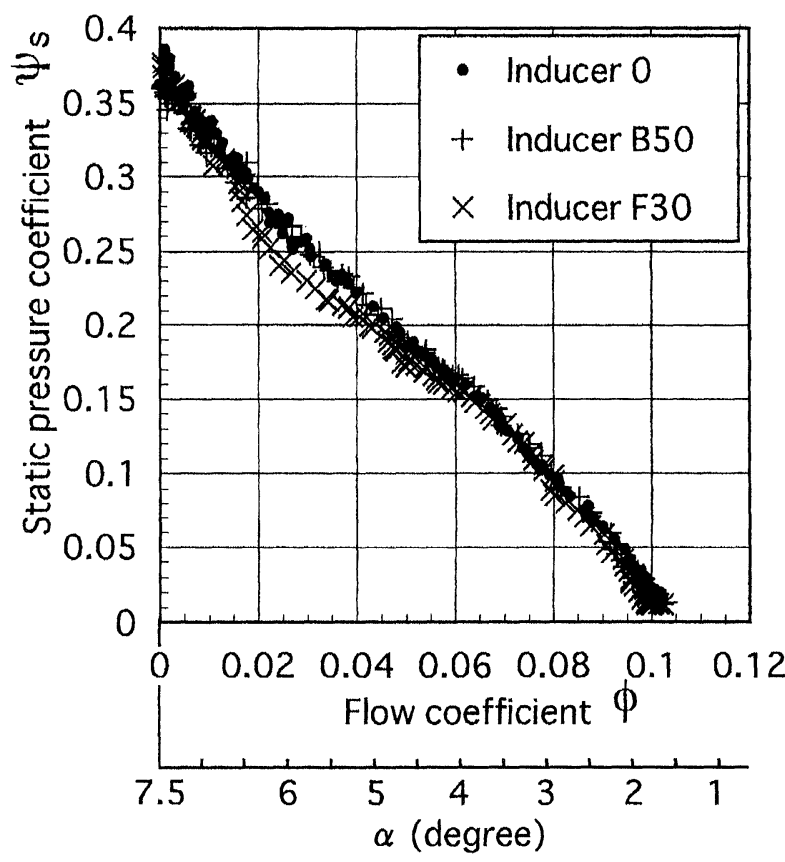

FIGURE 3

Non-cavitating performance of test inducers.

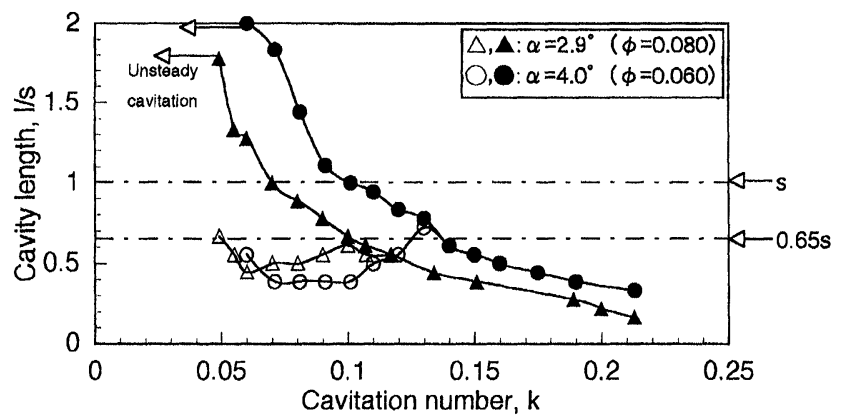

(a) Inducer 0

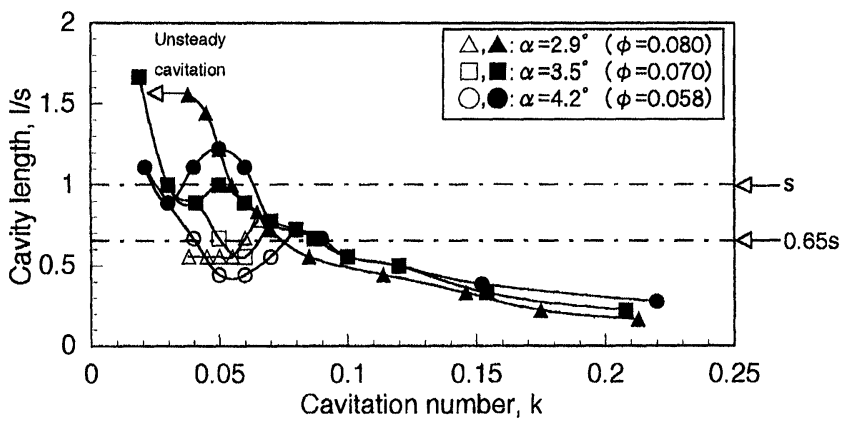

(b) Inducer B50

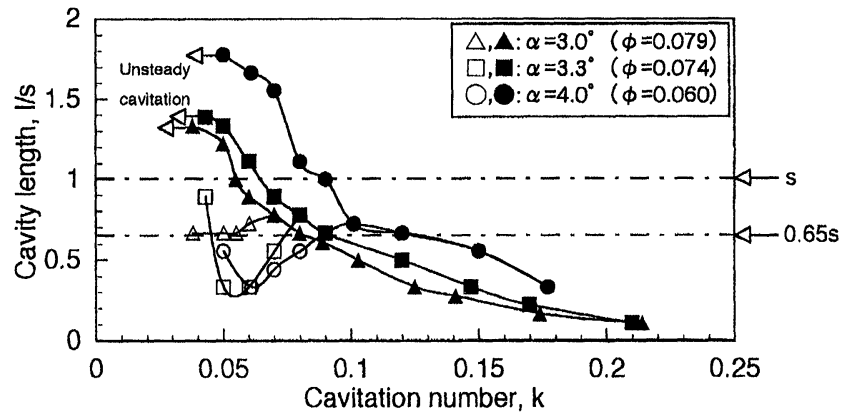

(c) Inducer F30

\section{FIGURE 4}

Plot of cavity length against $\mathrm{k}$. Open symbols show shorter cavity length for the case with alternate blade cavitation.

the root and then giving forward sweep by $25^{\circ}$. The tip/leading edge corner is rounded with radius $4 \mathrm{~mm}$. The leading edge curve is obtained by shifting the circumferential location of an involute curve with a base radius $26 \mathrm{~mm}$ (this produces a sweep with $85.3^{\circ}$ ) proportionally to the amount of sweep. The blade thickness is $2 \mathrm{~mm}$ and the suction surface near the leading edge is filed to wedge angle $2.75^{\circ}$ with the leading edge radius of $0.2 \mathrm{~mm}$.

Figure 3 shows the non-cavitating characteristics of the inducers. Nominal incidence angle at the tip $\left(\alpha=\beta-\tan ^{-1} \phi\right)$ is also shown. As expected, three inducers have nearly the same non-cavitating performance for $\phi>0.06(\alpha<4 \mathrm{deg})$. 
Figure 4 shows the plot of cavity length at the tip 1/s against the cavitation number $\mathrm{k}$ for the three inducers. For all inducers and all incidence angles shown, alternate blade cavitation (in which cavity length differs alternately) starts to develop when the cavity length exceeds about $65 \%$ of the spacing s. The cavitation becomes unsteady for the condition with $\mathrm{k}$ smaller than that with the data point. These observations agree fairly well with the theoretical findings by Horiguchi et al. (2000).

In most cases, unsteady cavitation starts to occur when the length of the shorter cavity exceeds $65 \%$ of the spacing. As expected, the cavity develops faster for the cases with a larger incidence angle $\alpha$.

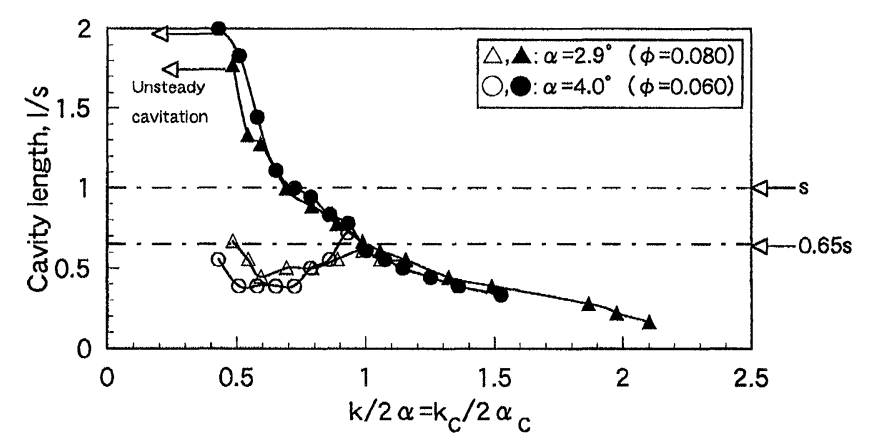

(a) Inducer 0

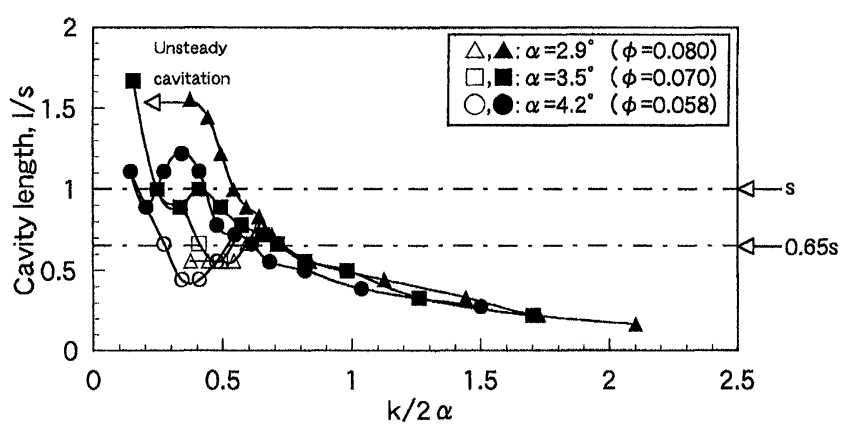

(b) Inducer B50

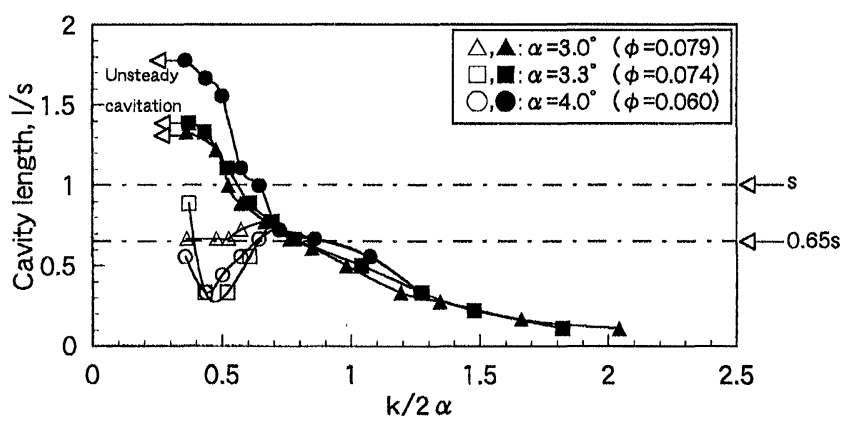

(c) Inducer F30

\section{FIGURE 5}

Plot of cavity length against $k / 2 \alpha$.
Figure 5 shows the plot of cavity length against $k / 2 \alpha$ where $\alpha$ is the nominal incidence angle at the tip. As expected from linearized analysis, the development of cavity is nearly the same for all the incidence angles. The comparisons among three inducers clearly show that the development of a steady cavity is significantly delayed by giving both forward and backward sweep.

Neglecting all the difference of the cascade geometry in the cross flow plane, the cavity length $1 / \mathrm{s}$ is replotted against $k_{c} / 2 \alpha_{c}$ in Fig. 6. Nominal values at the tip have been used for replotting. We find that the alternate blade cavitation starts to occur $k_{c} / 2 \alpha_{c} \approx 0.9$ and it shifts to unsteady cavitation at $k_{c} / 2 \alpha_{c} \approx 0.4$.

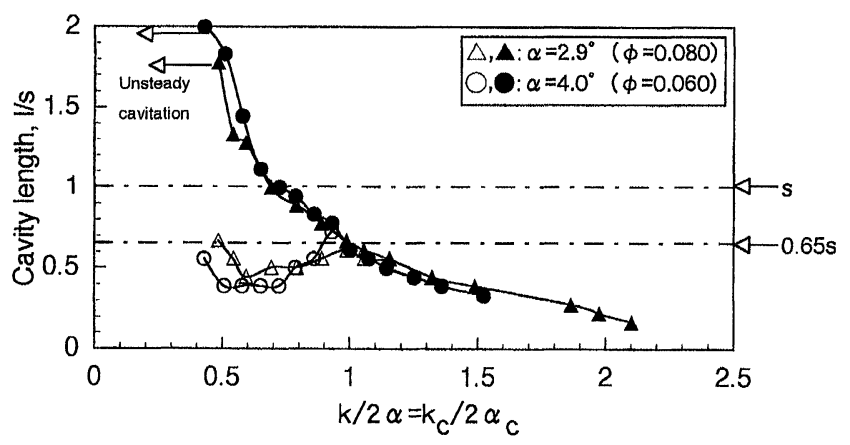

(a) Inducer 0

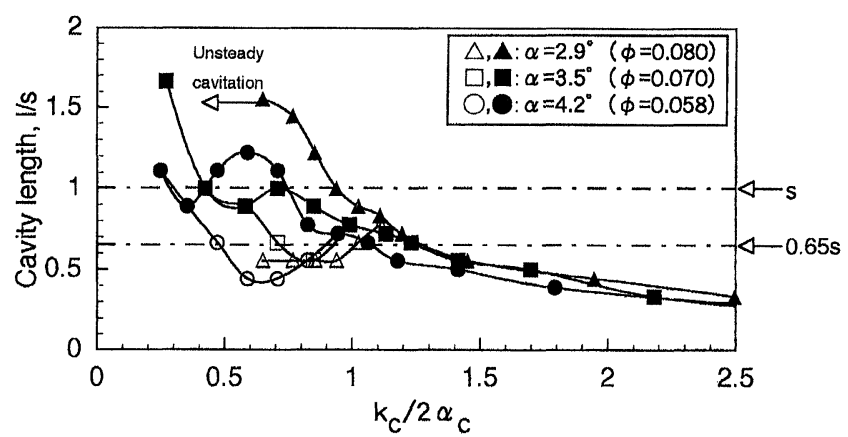

(b) Inducer B50

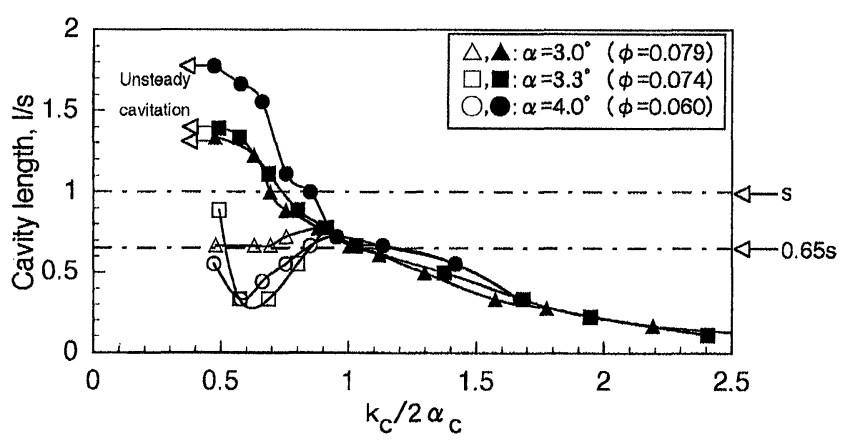

(c) Inducer F30

FIGURE 6

Plot of cavity length against $k_{c} / 2 \alpha_{c}$. 
TABLE II

Cascade parameters in physical and cross flow plane

\begin{tabular}{llll}
\hline \multicolumn{1}{c}{ Inducer } & \multicolumn{1}{c}{0} & \multicolumn{1}{c}{ B50 } & \multicolumn{1}{c}{ F30 } \\
\hline Sweep angle $\lambda$ at tip & $90 \mathrm{deg}$. & $35.4 \mathrm{deg}$. & $49.2 \mathrm{deg}$. \\
Inlet tip blade angle, $\beta_{t 1}$ & $7.5 \mathrm{deg}$. & $7.5 \mathrm{deg}$. & $7.5 \mathrm{deg}$. \\
Solidity at tip, C/s & 2.97 & 2.44 & 2.86 \\
Leading edge wedge angle, $\theta$ & $2.75 \mathrm{deg}$. & $2.75 \mathrm{deg}$. & $2.75 \mathrm{deg}$. \\
Effective blade angle, $\beta_{e}$ & $7.5 \mathrm{deg}$. & $12.8 \mathrm{deg}$. & $9.9 \mathrm{deg}$. \\
Effective solidity, $\mathrm{C}_{\mathrm{e}} / \mathrm{S}_{\mathrm{e}}$ & 2.97 & 2.40 & 2.84 \\
Effective leading edge wedge & $2.75 \mathrm{deg}$. & $4.74 \mathrm{deg}$. & $3.63 \mathrm{deg}$. \\
$\quad$ & & &
\end{tabular}

The present result shows that the delay of cavity development can be explained by the cross-flow effect. The secondary flow caused by the centrifuging of blade boundary layer should be quite different for forward and backward sweep. However, the delay of cavity development is quite the same for forward and backward sweep as shown in Fig. 5 and it can tbe explained by the cross-flow effect as shown in Fig. 6. This fact shows that the cross-flow effect is more important than the secondary-flow effect caused by fluid viscosity.

It has been shown (Tsujimoto et al., 1998) that various kind of unsteady cavitation depends only on the steady cavity length 1 or equivalently on $k_{c} / 2 \alpha_{c}$. In this respect, the present correction with $k_{c} / 2 \alpha_{c}$ explains not only the steady cavity development, but also the onset of unsteady cavitation for $k_{c} / 2 \alpha_{c}<0.4$.

Table II shows the cascade parameters in the cross flow plane. Comparisons in Fig. 6 have been made by neglecting the difference in the cascade geometry in the cross flow plane. To examine the effect of the geometrical difference, calculations are made by using a singularity method based on a linear closed cavity model (Horiguchi et al., 2000) on thin flat plate cascades. Figure 7 shows the cavity length in the cross flow plane. It is

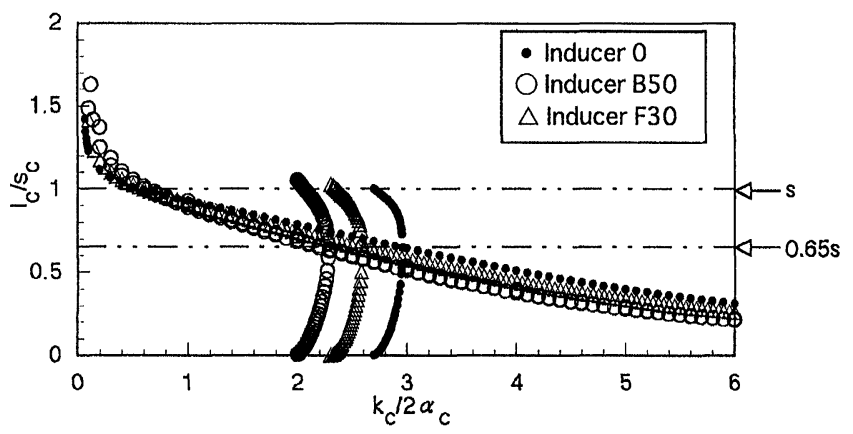

FIGURE 7

Calculated cavity length in cross flow plane. The results are shown for cross flow cascade geometries of Inducer 0, B50, F30. Two cavity lengths are shown where alternate blade cavitation was found. shown that the geometric effects also suppress the development of the cavity when the cavity is shorter than the spacing. Figure 8 compares the exact cavity length in the physical plane estimated from the exact cascade geometry in the cross flow plane with the approximate cavity length estimated from the original cascade geometry in the physical plane. Although the difference in the cascade geometry cannot be ignored, the major effect of sweep comes from the $k_{c} / 2 \alpha_{c}$ effect. Unfortunately the agreement with

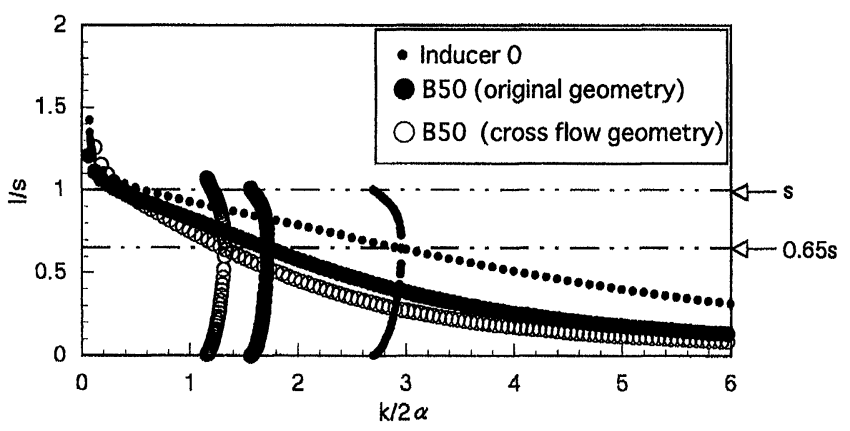

(a) Inducer 0 and Inducer B50

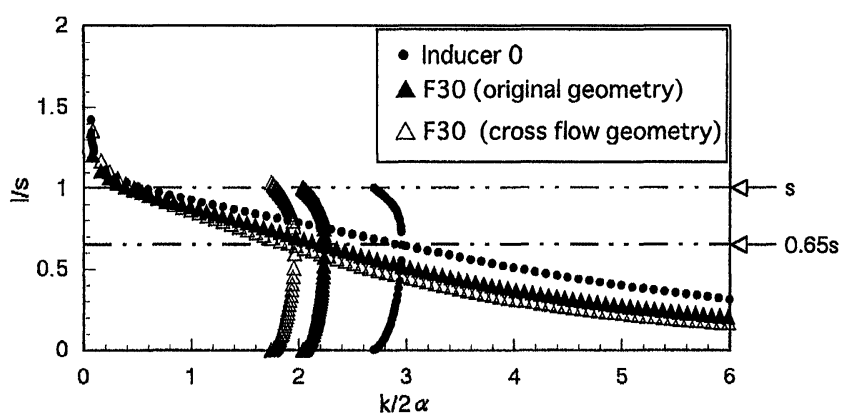

(b) Inducer 0 and Inducer F30

FIGURE 8

Calculated cavity length in physical plane, exact value from cross flow cascade geometry, and approximate value from original cascade geometry in physical plane. 


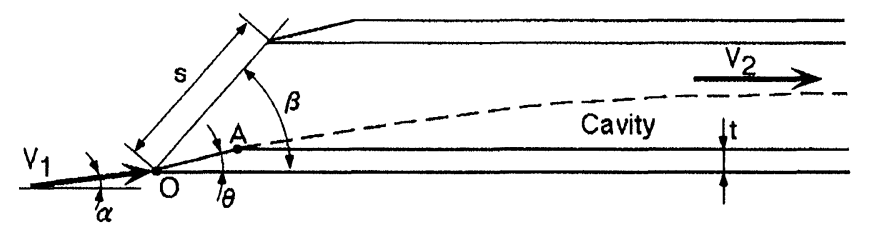

FIGURE 9

Geometry and the flow with wedged leading edge cascade.

the experiment in Fig. 5 is not good, perhaps caused by 3-D effects.

The results in Fig. 8 show that the sweep does not affect the cavity development largely for cavities longer than the spacing. This is caused by the canceling of the favorable effects of $k_{c} / 2 \alpha_{c}$ by the deteriorating effects of the cross-flow cascade geometry as shown in Fig. 7 for $1_{c} / s_{c}>1$. Analyses based on the correlation $k_{\text {choke }}=\alpha(\beta-\alpha)$ for the choke cavitation number of a thin-bladed cascade show that the sweep does not affect the choke cavitation number and is caused by the cancellation as mentioned.

To examine the effect on the choke cavitation number for the cascades with wedged leading edge cascade, calculations are made based on a linear theory by Acosta for wedged leading edge cascade as shown in Fig. 9. The cavity is assumed to start from the end of the leading edge wedge as shown in the figure. Figure 10 shows the choke cavitation number obtained. The results are not shown for a larger angle of attack for which the cavities will start from the leading edge tip. The choke cavitation number is clearly decreased by the sweep and the effect

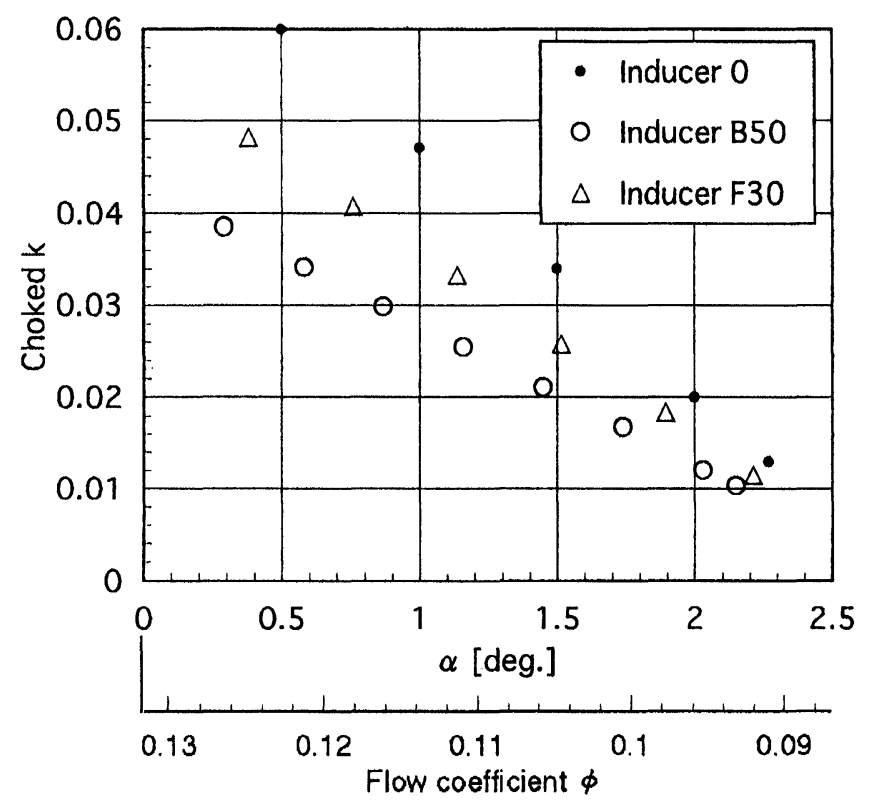

FIGURE 10

Choking cavitation number evaluated by an unpublished theory by Acosta for wedged leading edge cascade. is more significant for the cases with smaller angle of attack. This shows that the leading edge geometry significantly affects the cavity development. Unfortunately, the breakdown cavitation number could not be determined in the series of experiment due to the limitations of the experimental apparatus. Careful experiments are needed to determine the effect of sweep on the choke cavitation number, paying attention to the leading edge geometry and the location of the cavity detachment point.

\section{FURTHER REMARKS}

Applying this type of development to the kind of "sweep" one obtains by leaning back the blade elements from the typically radial orientation (thereby not changing the view from that of Inducer 0 in Fig. 1(a)-i.e., the projection of the leading edge of the leaned-back blade onto the end view of Fig. 1(a) is still a radial line) should reap the benefits on the breakdown value of cavitation number computed and plotted in Fig. 10. Adding a cut back to this geometry-in view of the development provided in this paper-may show further improvement.

\section{CONCLUSIONS}

1. A cross flow model is proposed to estimate the effect of leading-edge sweep on the cavity developments in inducer pumps with predominantly radial blade elements. Correlations are derived for geometrical parameters of the cascade in the cross-flow plane and for cavitation scaling.

2. The correlations are applied to the cases of forward and backward swept leading edges of inducers neglecting the detailed geometrical effects of the full impeller. Beneficial effects of both forward and backward sweep for cavitation are shown. The correlation using $k_{c} / 2 \alpha_{c}$ explains the cavity developments observed (e.g., cavity length, alternate blade cavitation, and unsteady cavitation) fairly well. This shows that the effect of sweep is mainly caused by the cross-flow effects proposed in the present paper with minor contributions of secondary (viscous) flow effects.

3. These effects of sweep are further studied with a linear closedcavity model in cascades without blade thickness. A favorable effect of sweep is found (causing shorter cavities) for cavities shorter than the blade spacing, but this effect disappears for longer cavities and in the choked cavity case.

4. The effect of a blunt leading edge blade shape, specifically a wedge, on the choked cavitation number is also examined with linear free streamline theory. Unlike the case of zero thickness blades, there is a favorable effect of sweep on the choked cavitation number which lowers the choked cavitation number and increases the resultant suction specific speed of the inducer pump. 
5. The analysis and results of this paper apply to blade "sweep" as generated largely by cutting back the leading edge of a typical radial-element-blade inducer. Similar and perhaps even greater improvements may be possible for leaned-back blade elements that are also cut back (at the leading edge) beyond this amount of lean.

\section{NOMENCLATURE}

$k \quad$ inlet cavitation number $=\left(p_{1}-p_{v}\right) /\left(\rho V_{1}^{2} / 2\right)$

$k_{c} \quad$ cross flow cavitation number

$=\left(p_{1}-p_{v}\right) /\left(\rho V_{\text {ctotal }}^{2} / 2\right)$

$p_{1} \quad$ static pressure upstream of impeller

$p_{v} \quad$ pressure in the cavity (presumed vapor)

$p_{t 1} \quad$ inlet total pressure

$s \quad$ spacing of blades along the cascade axis

$t \quad$ true blade thickness

$(t / s)_{\text {eff }}$ effective thickness/spacing ratio

$V_{1} \quad$ inlet relative velocity

$V_{\text {ctotal }}\left(V_{c}^{2}+V_{a}^{2}\right)^{1 / 2}$, total cross flow velocity in cross flow plane

$\alpha \quad$ angle between inlet relative velocity $V_{1}$ and the blade

$\beta \quad$ angle of blades from cascade axis

$\delta \quad$ angle from radial direction of leading edge in meridional plane leading edge in the meridional view $\lambda \quad$ true angle of blade leading edge-if $\lambda=\pi / 2$, leading edge is radial (In aerodynamic use the complement is called $\tau$.)

$\rho \quad$ liquid density

\section{REFERENCES}

Acosta, A. J., 1955, A Note on Partial Cavitation of Flat Plate Hydrofoils, Caltech Hydro Lab. Report No. E-19.9, Oct.

Cooper, P., 1973, High Flow Pump Impeller for Low Net Positive Suction Head and Method of Designing Same, United States Patent $3,737,249$.

Horiguchi, H., Watanabe, S., Tsujimoto, Y., and Aoki, M., 2000, A Theoretical Analysis of Alternate Blade Cavitation in Inducers, ASME, J. Fluids Engineering, vol. 122, pp. 156-163.

Ihara, A., Watanabe, H., and Shizukuishi, S., 1989, Experimental Research on the Effects of Sweep on Unsteady Hydrofoil Loadings in Cavitation, ASME, J. Fluids Engineering, vol. 111, pp. 263-270.

Sasaki, T., and Breugelmans, F., 1998, Comparison of Sweep and Dihedral Effects on Compressor Cascade Performance, ASME, J. Turbomachinery, vol. 120, pp. 454-464.

Tsujimoto, Y., Watanabe, S., and Horiguchi, H., 1998, Linear Analyses of Cavitation Instabilities of Hydrofoils and Cascade, US-Japan Seminar Abnormal Flows in Turbomachinery, Osaka, Japan.

Wadia, A. R., Szues, P. N., and Crall, D. W., 1998, Inner Workings of Aerodynamic Sweep, ASME, J. Turbomachinery, vol. 120 , pp. 671-683. 

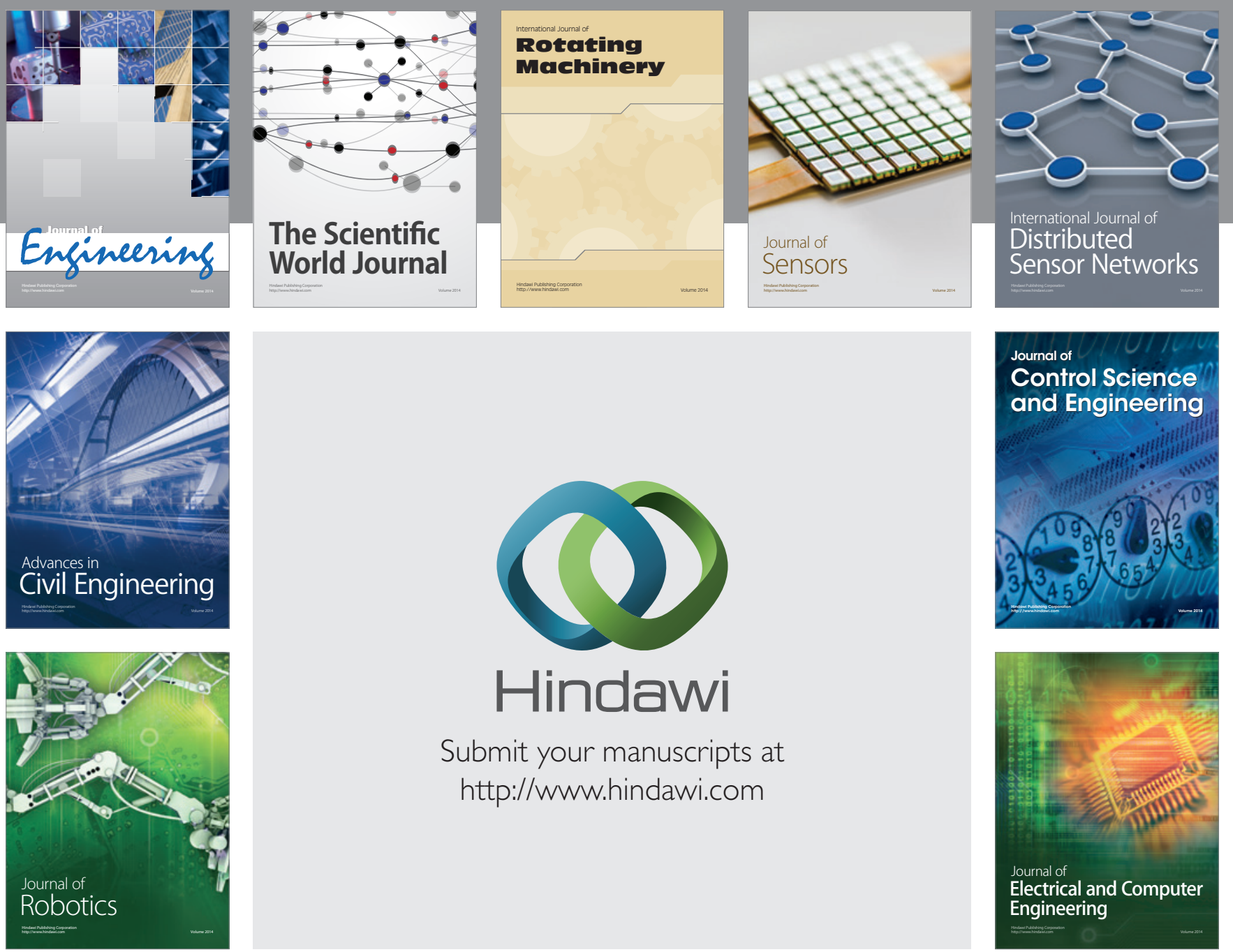

Submit your manuscripts at

http://www.hindawi.com
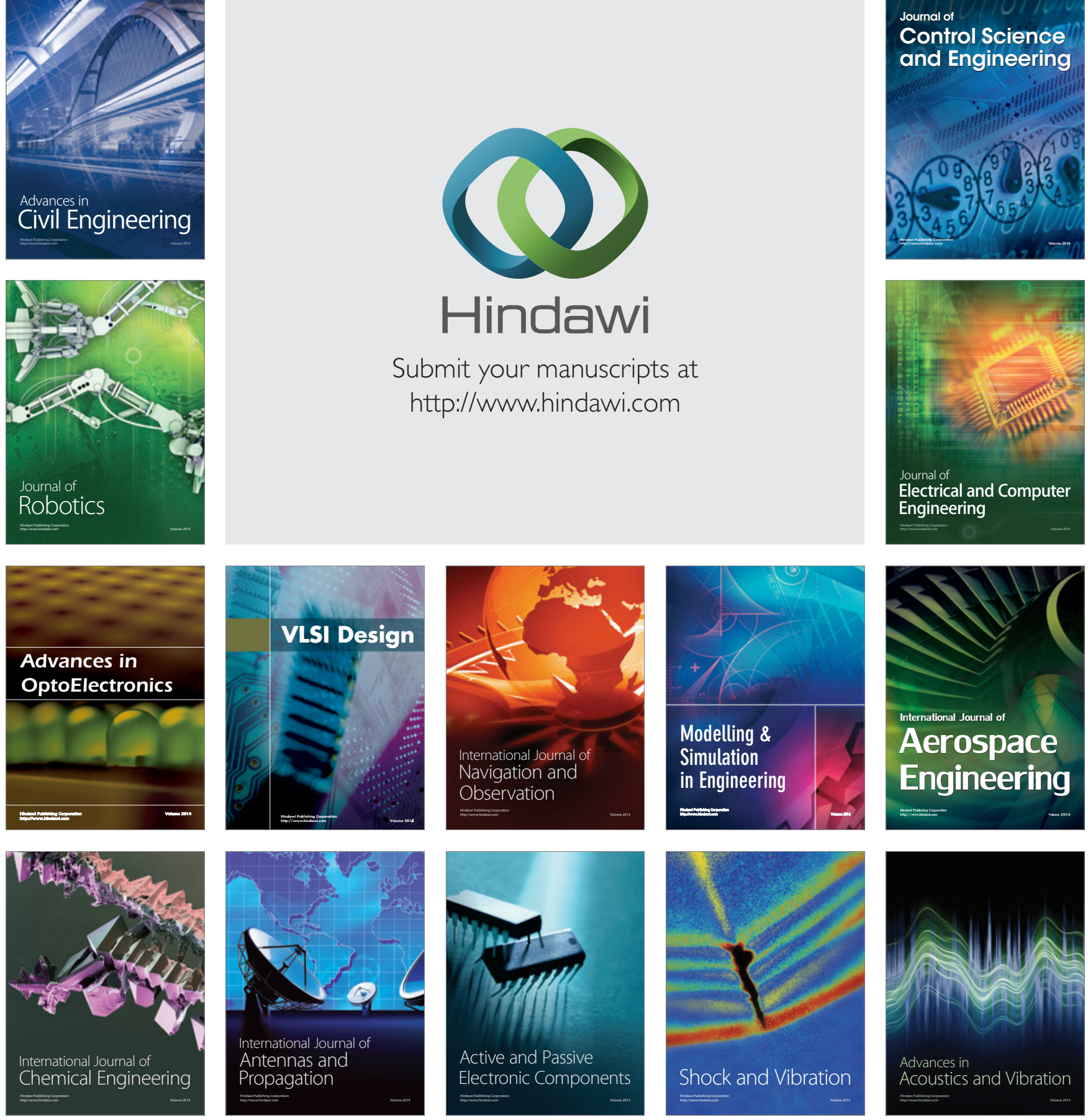